Correspondence: A. Bush, Dept of Paediatric Pulmonology, Royal Brompton Hospital and Imperial College, London, SW3 6NP, UK. E-mail: a.bush@rbh.thames.nhs.uk

\section{REFERENCES}

1 Barbato A, Bertuola F, Kuehni C, et al. Paediatrics in Berlin. Eur Respir J 2009; 34: 436-443.

\title{
Distinct clinical phenotypes of airways disease: a primary-care clinician perspective
}

\section{To the Editors:}

As a clinician involved in the diagnosis, treatment and aetiological research of obstructive airways disease in primary care practice-based networks, I read with interest the description by WEATHERALL et al. [1] of four or five distinct clinical types of airways disease. Their results appear to confirm and extend observations from an earlier population-based study that distinguished asthma and chronic airway obstruction, which was termed AS-CAO (analogous to their Cluster 1), from smoking-associated emphysematous chronic obstructive pulmonary disease (COPD) (Cluster 2) [2].

I would like to offer a personal perspective on the potential value to airway disease diagnosis, treatment and research, of this new taxonomy in the primary-care setting. In my experience AS-CAO and smoking-associated COPD represent two distinct clinical patterns of airways disease that can be easily recognised by an experienced clinician armed only with spirometry and home peak flow monitoring. The distinction is potentially important since AS-CAO and smoking-associated COPD may have different aetiologies and response to treatment [3]. Distinguishing Cluster 3 (classic atopic, eosinophilic asthma) from Cluster 4 (mild, nondescript disease) required further laboratory testing (serum immunoglobulin $\mathrm{E}$ and exhaled nitric oxide) not currently routinely used in primary care. Pending the results of further research, the importance of making this distinction in the primary-care setting is unclear to me. An important topic for future investigation is to describe the natural history of Cluster 4. Can Weatherall et al. [1] comment on the possibility that Cluster 4 subjects had had episodes of self-limited "acute asthmatic bronchitis" [4] that did not evolve into more severe forms of airways disease?

I agree with WEATHERALL et al. [1] that AS-CAO (Cluster 1) patients are a very ill group yet are excluded from both asthma and COPD studies. An ongoing clinical trial [5] was rejected for funding by the National Institute of Health because it aimed to enrol all eligible primary-care patients with reversible airways disease regardless of smoking status or other lung comorbidities (especially COPD). Until this dogma is overcome it is unlikely that progress will be made towards conducting long-term "real world" effectiveness trials in reversible airways obstructive disease(s) [6]. For these reasons I support adoption of this or a similar new taxonomy that: 1) is more congruent with the realities of airways disease than the current taxonomy; and 2) will enable successful implementation of effectiveness trials.

\section{Hahn}

Dean Medical Center, Family Practice, Madison, WI, USA.

Correspondence: D. Hahn, Dean East Clinic, 1821 S. Stoughton Road, Madison, WI 53716, USA. E-mail: dlhahn@wisc.edu

Statement of Interest: None declared.

\section{REFERENCES}

1 Weatherall M, Travers J, Shirtcliffe PM, et al. Distinct clinical phenotypes of airways disease defined by cluster analysis. Eur Respir J 2009; 34: 812-818.

2 Burrows B. Epidemiologic evidence for different types of chronic airflow obstruction. Am J Respir Dis 1991; 143: 1452-1455.

3 Hahn DL, Peeling RW. Airflow limitation, asthma, and Chlamydia pneumoniae-specific heat shock protein 60. Ann Allergy Asthma Immunol 2008; 101: 614-618.

4 Hahn DL. Acute asthmatic bronchitis: A new twist to an old problem. J Fam Pract 1994; 39: 431-435.

5 ClinicalTrials.gov AZMATICS: AZithroMycin/Asthma Trial In Community Settings. http://clinicaltrials.gov/show/NCT00266851.

6 Holgate S, Bisgaard H, Bjermer L, et al. The Brussels Declaration: the need for change in asthma management. Eur Respir J 2008; 32: 1433-1442.

DOI: $10.1183 / 09031936.00156709$

From the authors:

We thank D. Hahn for his interest in our paper [1]. We agree that following the natural history of individuals defined by these clusters may help define whether they represent distinct phenotypes, and we plan to do this in a follow-up cohort study of the Wellington Respiratory Survey. We agree that the fourth cluster identified by the "Diana" method may represent a phenotype of airway inflammation characterised by sputum production without marked elevation of exhaled nitric oxide and without marked airflow variability, as has been previously described [2,3]. However, we are cautious in extrapolating the results of the cluster analysis too far. The analysis was of a 
cross-sectional study and, although, for purposes of understanding, we presented the results in terms of the mean values of individual contributing variables, the cluster technique uses a multivariable distance measure in nine dimensions; thus, the associations between the contributing variables may be more complex than this. Apart from determining the natural history of these clusters in our cohort study, other forms of external validation, such as developing a clinical allocation rule and determining the response of different phenotypes to therapy, will also contribute understanding to the different phenotypes of chronic obstructive pulmonary disease.

\author{
M. Weatherall*, J. Travers", P. Shirtcliffe", S. Marsh", M. \\ Williams", M. Nowtiz ${ }^{\#}$ S. Aldington" and R. Beasley ${ }^{\#}$ \\ *Dept of Medicine, University of Otago, and "Medical Research \\ Institute of New Zealand, Wellington, New Zealand.
}

Correspondence: $\mathrm{M}$. Weatherall, Dept of Medicine, University of Otago, PB 7343, Wellington South, 6242, Wellington, New Zealand. E-mail: mark.weatherall@otago.ac.nz

Statement of Interest: None declared.

\section{REFERENCES}

1 Weatherall M, Travers J, Shirtcliffe $\mathrm{P}$, et al. Distinct clinical phenotypes of airways disease defined by cluster analysis. Eur Respir J 2009; 34: 812-818.

2 Wardlaw AJ, Silverman M, Siva R, et al. Multi-dimensional phenotyping: towards a new taxonomy for airway disease. Clin Exp Allergy 2005; 35: 1254-1262.

3 Haldar P, Pavord ID, Shaw DE, et al. Cluster analysis and clinical asthma phenotypes. Am J Respir Crit Care Med 2008; 178: 218-224.

DOI: 10.1183/09031936.00167309

\section{The Achilles heel of endothelin receptor therapy for pulmonary arterial hypertension}

\section{To the Editors:}

We were interested to read the letter from LAVELLE et al. [1], together with the accompanying editorial [2], regarding liver toxicity in patients receiving endothelin receptor antagonist therapy (ERA) for pulmonary arterial hypertension. Both of these articles highlight the potential for severe liver toxicity in patients receiving sitaxentan and stress the continued need for regular monitoring of liver function tests in all patients receiving ERAs. In fact, the details of the reported cases show that severe liver toxicity can occur within the time period of a month and one of us (P.A. Corris) has experience of a further case of fulminant hepatic failure occurring on treatment with sitaxentan when the liver function tests were normal less than one month beforehand. This suggests that all patients and the patients' relatives should be advised to report any symptoms or signs of hepatic disease, such as anorexia, abdominal pain or jaundice as soon as they are noticed.

We disagree with the suggestion that, based on current data, sitaxentan may be afflicted with a higher potential to severe liver toxicity. Severe toxicity has also been reported with bosentan, with or without other potential medication interactions.

NAGAi et al. [3] reported multi organ failure following severe hepatitis (AST and ALT levels >5000 IU) in a patient receiving bosentan. That patient died. DWYER et al. [4] reported severe toxicity with the combination of bosentan and methotrexate, which did not recur on the presence of methotrexate alone. One of us (P.A. Corris) has recently diagnosed a case of severe liver toxicity due to hypersensitivity reaction to ambrisentan. HUMBERT et al. [5] reported on nine patients satisfying Hy's law for severe liver toxicity due to bosentan therapy on the TRAX safety database, though no clinical details were reported. About $20 \%$ of patients treated with bosentan in Europe were not entered into that database, so there may have been more cases. Under-reporting of all medication-related complications is a chronic health care issue.

In summary, physicians should be aware that severe hepatic toxicity may be seen in patients receiving any of the current ERA therapies, and that there may be interactions with other potentially hepatotoxic medications. Patients must be advised to consult their prescribing physician urgently on the first signs and symptoms of hepatic toxicity, irrespective of normal liver function tests within a month earlier. Every case of severe hepatotoxicity should be reported. Knowing the true degree of vulnerability of this particular "Achilles' heel" will allow for better scientific conclusions.

\section{P.A. Corris* and D. Langleben ${ }^{\#}$}

*Institute of Cellular Medicine Newcastle University and Cardiothoracic Centre Freeman Hospital Newcastle, UK. \#Center for Pulmonary Vascular Disease, Jewish General Hospital, Montreal, QC, Canada.

Correspondence: P.A. Corris, Institute of Cellular Medicine Newcastle University and Cardiothoracic Centre, Freeman Hospital, Newcastle NE7 7DN, UK. E-mail: paul.corris@ncl.ac.uk

Statement of Interest: Statements of interest for both authors of this manuscript can be found at www.erj.ersjournals.com/misc/ statements.dtl 